\title{
Characterization and Chemical Stability of Hydrophilic and Hydrophobic Magnetic Nanoparticles
}

\author{
Natália Cristina Candian Lobato a, Marcelo Borges Mansur ${ }^{a, c, d}$, Angela de Mello Ferreira ${ }^{b, d *}$ \\ ${ }^{a}$ Postgraduate Program on Metallurgical, Materials and Mining Engineering, Universidade Federal de \\ Minas Gerais (UFMG), Belo Horizonte, MG, Brazil \\ ${ }^{b}$ Department of Chemistry, Centro Federal de Educação Tecnológica de Minas Gerais (CEFET-MG), \\ Belo Horizonte, MG, Brazil \\ ${ }^{c}$ Program of Metallurgical and Materials Engineering, Universidade Federal do Rio de Janeiro \\ (UFRJ), Rio de Janeiro, RJ, Brazil \\ ${ }^{d}$ National Institute of Science and Technology on Mineral Resources, Water and Biodiversity (INCT-Acqua), \\ Brazil
}

Received: September 22, 2016; Revised: March 06, 2017; Accepted: March 11, 2017

\begin{abstract}
Magnetic nanoparticles can improve the efficiency of phase separation time in multi-stage operations when a magnetic field is present. As such operations involve contact with aqueous and/or organic solutions, hydrophilic magnetic nanoparticles synthesized through the co-precipitation method were functionalized with oleic acid to attain hydrophobic magnetic nanoparticles. Both nanoparticles were characterized morphologically, chemically and magnetically. The results revealed that the particles (size $\approx 10 \mathrm{~nm}$ ) consisted of an iron oxide mixture of magnetite and maghemite. The functionalization with oleic acid was effective in converting them into hydrophobic nanoparticles. Both particles were ferro/ferrimagnetic and the presence of oleic acid did not interfere significantly in the saturation magnetization value. The chemical stability of both nanoparticles were also evaluated, as an attempt of simulating broad industrial conditions to which the nanoparticles may be subjected; the hydrophilic nanoparticles were resistant at $\mathrm{pH} \geq 4$, while the hydrophobic nanoparticles were stable at $\mathrm{pH} \geq 3$.
\end{abstract}

Keywords: Magnetic Nanoparticles, Chemical Stability, Oleic Acid, Hydrophilic Nanoparticles, Hydrophobic Nanoparticles

\section{Introduction}

The use of magnetic nanoparticles to develop and/or improve separation techniques has been widely studied in recent years. In general, the separation methods with nanoparticles have proven to be fast, easily automatable, reversible, and selective, in addition to being able to reach high efficiency levels ${ }^{1-3}$. Magnetic nanoparticles have been used as contrast agents in diagnostic applications, drug release, magnetic refrigeration, magnetic separation, among others ${ }^{46}$. In the field of mineral processing, the application of nanoparticles has been studied in multi-stage operations, such as solvent extraction ${ }^{1,7-10}$, adsorption ${ }^{11-15}$, and ion exchange ${ }^{16,17}$.

In solvent extraction, the application of magnetic nanoparticles is intended to magnetize the organic solution to make the separation between the aqueous and the organic phases quick and efficient when in the presence of a magnetic field ${ }^{7,18}$. To obtain a magnetized organic solution, hydrophobic superparamagnetic nanoparticles are dispersed in an organic solvent containing an extractant, responsible to selectively react with the metal in the aqueous phase. After contact with the two phases, and the metal thus being transferred from

\footnotetext{
* e-mail: angelamello@des.cefetmg.br
}

the aqueous phase to the organic phase, a magnetic field is applied to accelerate the phase disengagement step ${ }^{1,7-10}$. In fact, the results of phase disengagement with magnetic nanoparticles was faster, but the values varied significantly depending on the operating conditions and other specification of the system, such as the type and the concentration of the extractant, aqueous composition, concentration of the nanoparticles, etc. According to Palyska and Chmielewski ${ }^{8}$, the use of magnetic separation in solvent extraction can achieve a separation rate 160 times faster than using the gravitational field alone; Vatta, Koch, and Sole ${ }^{9}$ reported that the time separation when using the magnetic system is between $48 \%$ and $86 \%$ when compared to the traditional system time; and Lobato, Ferreira, and Mansur ${ }^{10}$ verified that magnetic separation is 3-5 times faster when using magnetic fluid.

The superparamagnetic nanoparticles are also being studied for metal ion adsorption from an aqueous solution ${ }^{11-15}$. Similar to in solvent extraction, the use of magnetic properties in the adsorbent material is intended to improve the solid-liquid separation processes after metal loading processes occur. Moreover, because of the small size of the nanoparticles, the adsorption process is favored due to the large surface area. The magnetic nanoparticles are used as a core and their surface is modified in order to achieve 
selectivity for a given substance or metal present in the aqueous phase. Once loaded, the magnetic particles can easily be separated from the depleted solution by using a magnetic field. Such operation may reduce the process time and increase the efficiency of the phase separation. Thus, the loaded particles can be regenerated and reused 13-15. Magnetic nanoadsorbents have proved efficient for ions such as $\mathrm{Cd}(\mathrm{II}), \mathrm{Zn}$ (II), $\mathrm{Pb}$ (II), $\mathrm{Cu}$ (II) ${ }^{11}, \mathrm{As}$ (III) ${ }^{12}, \mathrm{P}^{13}$, $\mathrm{Cr}(\mathrm{IV}){ }^{14}$, and $\mathrm{Sb}$ (III) ${ }^{15}$.

Also aimed at a faster and more efficient solid-liquid separation, magnetic particles have been proposed in the ion-exchange process ${ }^{16,17}$. The technique is very similar to the adsorption process. Modified magnetic nanoparticles capable of performing ion exchange are dispersed in an aqueous solution (or an effluent) containing the element to be removed. The loaded nanoparticles are separated from the aqueous phase through the presence of a magnetic field. Subsequently, ions are removed from the particles, which are regenerated and reused. This method has several advantages over the traditional method. First, problems such as clogging and fouling do not occur. Second, because of the small particle size, there is a large surface area for ion exchange reactions. And finally, there is the benefit of magnetic separation, which is faster than sedimentation or filtration and more selective, since only the magnetic fraction is separated from the aqueous phase ${ }^{16}$.

Given the prospect of using magnetic nanoparticles in the mining industry, this work aims to characterize hydrophilic and hydrophobic magnetic nanoparticles. Hydrophilic magnetic nanoparticles were synthesized by the co-precipitation method with no surface modification, while the hydrophobic nanoparticles were obtained by functionalizing the hydrophilic nanoparticles with oleic acid. Oleic acid $\left(\mathrm{C}_{18} \mathrm{H}_{34} \mathrm{O}_{2}\right)$ is an organic species with high affinity to the surface of ferrous oxides due to its carboxylic group, and because of this, it is often used as a surfactant to modify the surface of magnetite nanoparticles, making them hydrophobic. The functionalization process with oleic acid is considered to be simple and inexpensive ${ }^{5,19,20}$. The nanoparticles were characterized for their morphology, composition, and magnetization. Furthermore, the stability of the particles when in contact with aqueous solutions of varying acidity was also evaluated, in an attempt to simulate a number of industrial conditions.

\section{Experimental}

\subsection{Materials}

Ferrous sulphate heptahydrate $\left(\mathrm{FeSO}_{4} \cdot 7 \mathrm{H}_{2} \mathrm{O}\right.$, Neon, purity $99 \%$ ), ferric chloride hexahydrate $\left(\mathrm{FeCl}_{3} \cdot 6 \mathrm{H}_{2} \mathrm{O}\right.$, Vetec, purity $97 \%$ ), ammonium hydroxide $\left(\mathrm{NH}_{4} \mathrm{OH}, \mathrm{Neon}, 28-30\right.$ wt.\%), oleic acid $\left(\mathrm{C}_{18} \mathrm{H}_{34} \mathrm{O}_{2}\right.$, Synth, purity $\left.100 \%\right)$, ethyl alcohol $\left(\mathrm{CH}_{3} \mathrm{CH}_{2} \mathrm{OH}\right.$, Neon, purity $\left.99.5 \%\right)$, sulfuric acid $\left(\mathrm{H}_{2} \mathrm{SO}_{4}\right.$, Synth, purity $\left.98 \%\right)$, potassium nitrate $\left(\mathrm{KNO}_{3}\right.$, Vetec, purity 99\%), and Exxsol D80 (liquid aliphatic hydrocarbon,
ExxonMobil Chemical). Except for the organic diluent, which was of commercial grade, all remaining reagents used in this work were of analytical grade, and the water was distilled or Milli-Q (Millipore, France), depending on the experiment.

\subsection{Synthesis of the hydrophilic magnetic nanoparticles}

The nanoparticles were obtained by the co-precipitation method ${ }^{5,21-24}$. In $300 \mathrm{~mL}$ of distilled water, $19.7 \mathrm{~g}$ of $\mathrm{FeSO}_{4} \cdot 7 \mathrm{H}_{2} \mathrm{O}$ and $39.0 \mathrm{~g}$ of $\mathrm{FeCl}_{3} \cdot 6 \mathrm{H}_{2} \mathrm{O}$ were dissolved (molar ratio of ferric ion to ferrous ion in the solution of two). The solution was vigorously stirred (1000 rpm) and heated to $80{ }^{\circ} \mathrm{C}$. Then, $50 \mathrm{~mL}$ of $\mathrm{NH}_{4} \mathrm{OH}$ were dropped under stirring, and the solution continued to be stirred for 40 minutes. After, heating and stirring were turned off and the solution rested until reaching room temperature. The magnetic nanoparticles were washed with distilled water and then with ethyl alcohol. After that, they were filtrated, and dried in a kiln $\left(60^{\circ} \mathrm{C}\right)$ for 2 hours.

\subsection{Synthesis of hydrophobic magnetic nanoparticles}

The hydrophilic magnetic nanoparticles were functionalized with oleic acid in order to make them hydrophobic ${ }^{7}$. In a $100 \mathrm{~mL}$ glass reactor, $1 \mathrm{~g}$ of the synthesized magnetic nanoparticles was dispersed in $40 \mathrm{~mL}$ of distilled water at $60{ }^{\circ} \mathrm{C}$ under mechanical stirring $(500 \mathrm{rpm})$. Next, $1 \mathrm{~mL}$ of oleic acid was added into the solution and the mixture was stirred for 15 minutes. The solution rested until reaching room temperature. The hydrophobic particles were removed using a magnet, washed with distilled water and then with ethyl alcohol, filtrated, and dried in a kiln $\left(60^{\circ} \mathrm{C}\right)$ for 1 hour.

\subsection{Characterization of the magnetic nanoparticles}

The magnetic nanoparticles were characterized using the following methods:

(i) Transmission electron microscopy (TEM) images were obtained in order to analyze the morphology structure and measure the particle size distribution of the nanoparticles. The analysis was performed using a Tecnai G2-20 equipment, SuperTwin FEI (200 kV);

(ii) The surface area of the nanoparticles was determined by the BET (Brunauer-Emmett-Teller) method through nitrogen adsorption using a Quantachrome Nova 1200e.

(iii) X-ray diffraction (XRD) was used to evaluate the crystallography of the nanoparticles. Tests were carried out using a Shimadzu 7000 X-ray diffractometer. The $\mathrm{CuK} \alpha$ radiation source was operated at $40 \mathrm{kV} / 30 \mathrm{~mA}$ using a graphite crystal monochromator. The scan 2 theta ranged 
from 10 to 80 , with increments of 0.02 theta and a scanning speed of $2^{\circ} \mathrm{min}^{-1}$;

(iv) Raman spectroscopy was used to differentiate the iron oxide phases (magnetite and maghemite). The technique was performed using a Jobin Yvon Horiba LABRAM equipment, HR800 model, with a He-Ne laser of $632.8 \mathrm{~nm}$, coupled to an Olympus BX-41 microscope. The spectra were acquired with a laser power $0.08 \mathrm{~mW}$ in the range of $150-900 \mathrm{~cm}^{-1}$;

(v) Broad sextets and model-independent hyperfine field distribution were collected with the sample at $150 \mathrm{~K}$ and 298 $\mathrm{K}$ (in a liquid helium bath-cryostat) in a constant acceleration transmission mode setup with a $20 \mathrm{mCi}-57 \mathrm{Co} / \mathrm{Rh}$ source. Data were numerically fitted by Lorentzian functions with the least-square procedure of the NORMOS ${ }^{\mathrm{TM}}$ program (Brand RA, Laboratorium für Angewandte Physik, Universität Duisburg, D-47048, Duisburg, Germany). The isomer shift values were referred to $\alpha-\mathrm{Fe}$ at room temperature (RT);

(vi) The zeta potential of the hydrophilic nanoparticle was measured in order to compare the electrostatic potential of the surface of the synthesized nanoparticle with values reported in the literature. For sample preparation, $0.2 \mathrm{~g}$ of nanoparticles were added to $200 \mathrm{~mL}$ of $0.001 \mathrm{~mol} / \mathrm{L} \mathrm{KNO}_{3}$, and the $\mathrm{pH}$ was adjusted between 5 and 10, using $\mathrm{NH}_{4} \mathrm{OH}$ and $\mathrm{H}_{2} \mathrm{SO}_{4}$ solutions. Each sample was analyzed by a Zeta Meter device (ZD3-D-G 3.0+ model), collecting at least 10 PZC values;

(vii) The adsorption of the oleic acid in the magnetic nanoparticles was verified by Fourier transform infrared spectroscopy (FTIR), which identified the chemical groups of the hydrophilic nanoparticles and the functional groups of the oleic acid. The analyses were performed in the range of 4000 to $450 \mathrm{~cm}^{-1}$ using a Perkin Elmer infrared spectrophotometer (Spectrum Frontier model);

(viii) Thermogravimetric analysis (TGA) was used to evaluate the thermal stability of the magnetic particles, as well as to check the amount of oleic acid adsorbed on the functionalized nanoparticles. The experiment was carried out using a Perkin Elmer STA 6000 equipment and the procedure was performed in nitrogen atmosphere at a flow rate of 20 $\mathrm{mL} /$ minute, and under the temperatures scan from $30^{\circ} \mathrm{C}$ and $800{ }^{\circ} \mathrm{C}$, at a heating rate of $10^{\circ} \mathrm{C} /$ minute;

(ix) The magnetic characteristics and behavior of the nanoparticles were obtained using the Vibrating Sample Magnetometer (VSM), Lakeshore model 7404 series. The hysteresis loops were measured under a magnetic field strength of 11500 Gauss at room temperature;

(x) The chemical stability of the hydrophilic and hydrophobic nanoparticles was determined by contacting them with water at changing acidity. Regarding the hydrophilic magnetic nanoparticles, $0.25 \mathrm{~g}$ of the material was added to $25 \mathrm{~mL}$ of Milli-Q water (Millipore, France) at changing acidity $\left(\left[\mathrm{H}^{+}\right]=10^{-7}, 10^{-6}, 10^{-5}, 10^{-4}, 10^{-3}, 10^{-2}, 10^{-1}, 0.5,1\right.$, and $2 \mathrm{~mol} / \mathrm{L}$ ) in Erlenmeyer flasks. The water was acidified using $\mathrm{H}_{2} \mathrm{SO}_{4}$ solutions. The hydrophobic magnetic nanoparticles were dispersed in Exxsol D80, a common diluent used in the industrial solvent extraction processes, at a concentration of $10 \mathrm{~g} / \mathrm{L}$, using an ultrasound bath (Brasonic, 1210 model, frequency $47 \mathrm{~Hz}$ ) for 30 minutes. After the formation of the magnetic fluid or ferrofluid, $25 \mathrm{~mL}$ of the organic liquid was placed in contact with $25 \mathrm{~mL}$ of Milli-Q water at changing acidity $\left(\left[\mathrm{H}^{+}\right]=10^{-7}, 10^{-6}, 10^{-5}, 10^{-4}, 10^{-3}, 10^{-2}, 10^{-1}, 0.5,1\right.$, and $2 \mathrm{~mol} / \mathrm{L}$ ) in Erlenmeyer flasks. All the samples were stirred in a shaker (New Brunswick Scientific, Annova44 model) at $400 \mathrm{rpm}$, for 24 hours, at room temperature $\left(25^{\circ} \mathrm{C}\right)$. After, a sample of the water was withdrawn for chemical analysis by Atomic Absorption Spectroscopy (GBC, XplorAA-2 model) to determine the content of iron. Such tests were performed in triplicate.

\section{Results and Discussion}

The morphology and size distribution of the nanoparticles were obtained via transmission electron microscopy (TEM). The images shown in Figure 1 revealed that the synthesized nanoparticles have a nearly spherical shape, with homogeneous distribution. Hydrophilic nanoparticles are shown in Figure 1 - A and B, while hydrophobic nanoparticles are shown in Figure 1 - C and D. The layer of oleic acid adsorbed on the surface of the magnetic hydrophobic nanoparticles is shown to surround the particles. The hydrophilic magnetic nanoparticles have sizes ranging between 6 and $16 \mathrm{~nm}$, with a mean diameter of $10.2 \mathrm{~nm}$, while the hydrophobic magnetic nanoparticles, functionalized with oleic acid, have sizes ranging between 7 and $18 \mathrm{~nm}$, with a mean diameter of $11.0 \mathrm{~nm}$. The values of the diameters were calculated using the software Image J, with more than 300 values measured for each sample. Size histograms of both nanoparticles are shown in Figure 2. The distribution profile of the values of diameters follows the log-normal function, as had been observed in a previous study ${ }^{25}$. Based on such results, it can be concluded that the functionalization of the magnetic nanoparticles with oleic acid did not modify them regarding their morphology and size.

The surface area was determined by the BET method. The values of the hydrophilic and hydrophobic nanoparticles were 71.6 and $55.3 \mathrm{~m}^{2} / \mathrm{g}$, respectively. Both materials present large surface areas. Such character is desirable in order to enhance a higher mass transfer and chemical reaction rates on adsorption and ion exchange processes, in the case of the hydrophilic nanoparticles; and it is favorable to improve the distribution of the nanoparticles in the bulk of the organic phase on the solvent extraction process, in the case of the hydrophobic nanoparticles.

X-ray diffraction revealed the crystalline structure of the nanoparticles (Figure 3). The materials showed well defined peaks at (111), (220), (311), (400), (422), (511), (440), (620), and (533), which correspond to an inverse cubic 

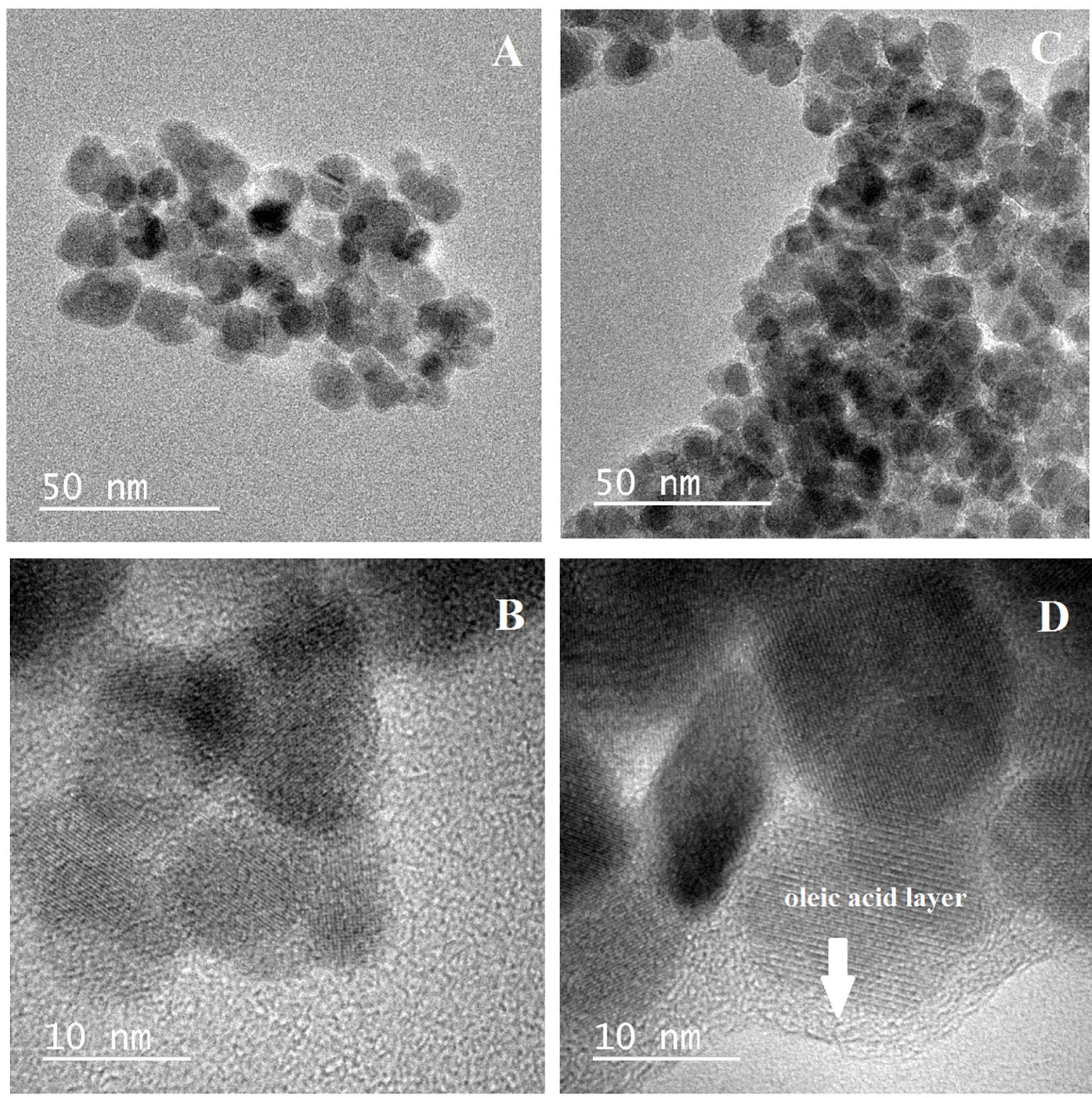

Figure 1. Transmission electronic microscopy images of the hydrophilic nanoparticles (A and B) and the hydrophobic nanoparticles functionalized with oleic acid (C and D).

spinel structure. This structure is characteristic of magnetite $\left(\mathrm{Fe}_{3} \mathrm{O}_{4}\right)$ or maghemite $\left(\gamma-\mathrm{Fe}_{2} \mathrm{O}_{3}\right)$, not being possible to identify which of the phases is present or if both phases are present in the material. The diffractograms of hydrophilic and hydrophobic nanoparticles are similar, indicating that the functionalization process with oleic acid did not modify the crystallography character of the magnetic nanoparticles.

The crystallite size of particles can be estimated by the Scherrer's equation:

$$
D=\frac{K \lambda}{\beta \cdot \cos \theta}
$$

where $\mathrm{K}$ is the Scherrer constant, whose value is 0.9 for spherical crystals, $\lambda$ is the $\mathrm{X}$-ray wave length, $\beta$ is the half-width of the maximum diffraction peak expressed in radians and $\theta$ is the diffraction angle of the peak position ${ }^{23,26}$ The application of the Scherrer's equation to the peak (311) with $2 \theta \sim 35.7^{\circ}$ of the magnetic nanoparticles indicated that the crystalline size of hydrophilic and hydrophobic nanoparticles were of 11.2 and $12.1 \mathrm{~nm}$, respectively. The calculated sizes are according to the average sizes found via transmission electron microscopy (TEM). Moreover, the results found by Scherrer's equation are statistically similar, because the crystalline cores of both samples come from the exact same synthesis. In addition, the oleic acid present in the hydrophobic particles is not detected by the X-ray diffraction method (it has no crystallographic organization), therefore it can not influence on the crystallite size calculated by Scherrer's equation. 


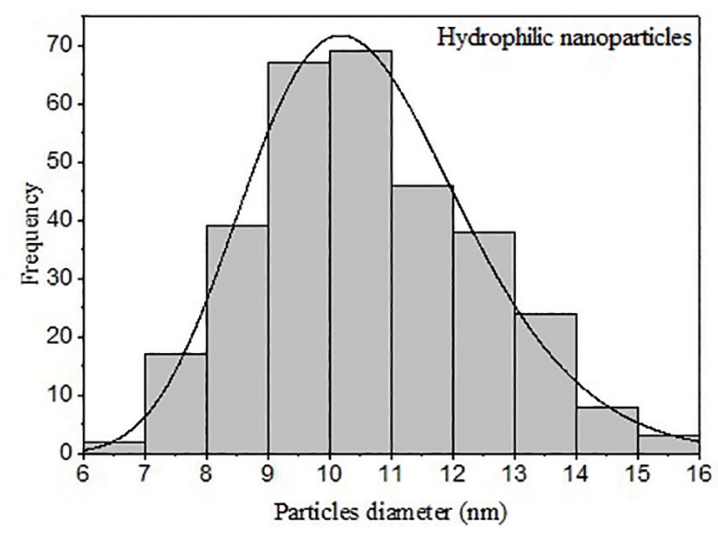

A

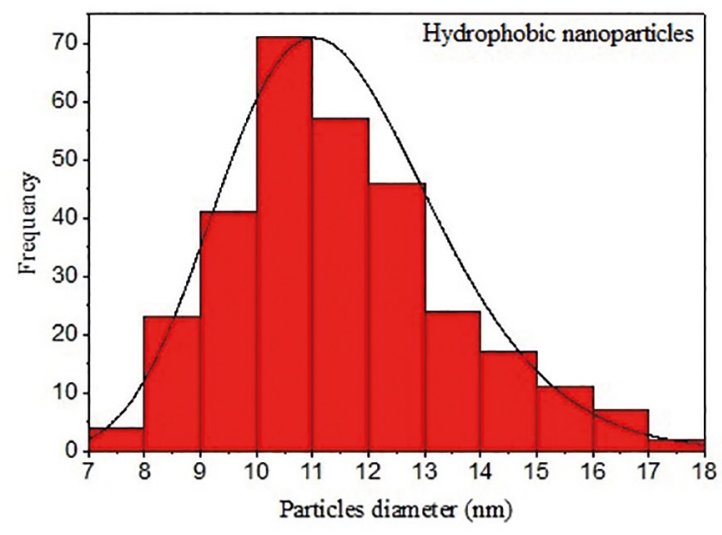

B

Figure 2. Size distribution histogram of the hydrophilic nanoparticles (A) and the hydrophobic nanoparticles (B).

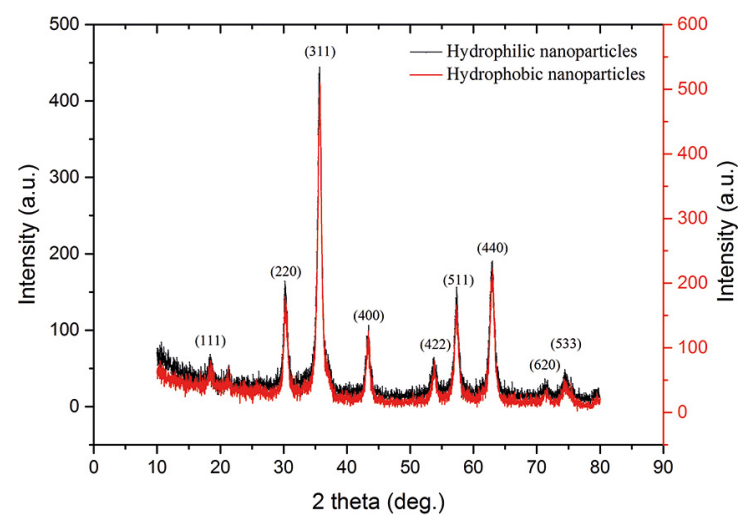

Figure 3. X-ray diffractograms of the magnetic nanoparticles.

Raman spectroscopy analysis complements X-ray diffractograms, since it differentiates distinct phases of iron oxide present in the sample, such as magnetite $\left(\mathrm{Fe}_{3} \mathrm{O}_{4}\right)$ and maghemite $\left(\gamma-\mathrm{Fe}_{2} \mathrm{O}_{3}\right)$. Under the microscope linked to the equipment, it was observed that the samples of hydrophilic and hydrophobic nanoparticles presented areas with distinct colors, one brown and another black. For this reason, the analyses were performed on each distinct area of both samples, the results of which are shown in Figure 4. The vibrational modes of magnetite and maghemite structures in Raman spectroscopy are summarized in Table 1. A fitting of the peaks of magnetite and maghemite spectra was performed via PeakFit function of the Origin software, which it can be seen in Figure 4. Analyzing the characteristic peaks of each phase, it can be concluded that magnetite and maghemite phases occur in both magnetic nanoparticles, and that the black area corresponds to the predominance of the magnetite phase, while the brown area is related to the predominance of the maghemite phase.

The occurrence of magnetite and maghemite was also identified from the Mössbauer spectroscopy data for the samples at $150 \mathrm{~K}$ (Table 2 and Figure 5). Both $298 \mathrm{~K}$ spectra show broad line patterns with some small differences in their six line shapes. The broad six lines of the $298 \mathrm{~K}$ spectra of both samples can be attributed to the finite-size effect and/ or spin relaxation of the analyzed nanoparticles. However, no superparamagnetic state (absence of doublet component) has been observed probably due to the presence of particles with a larger size indicated by XRD and TEM data and/or due to the intra-particle magnetic interactions. Therefore, in order to better quantify the phases, measurements at 150 $\mathrm{K}$ were performed. This temperature level is above the Verwey temperature, $\mathrm{T}_{\mathrm{V}} \sim 121 \mathrm{~K}$, which is the temperature that the magnetic conductivity is significantly reduced and the magnetic behavior changes completely ${ }^{30}$. Each spectra obtained at $150 \mathrm{~K}$ was fitted with three sextets with linewidth $\sim 0.50 \mathrm{~mm} / \mathrm{s}$. The sextet with the largest hyperfine magnetic field (Table 2) was assigned to $\mathrm{Fe}^{3+}$ in maghemite. The other two sextets were assigned to $\mathrm{Fe}^{3+}$ in the tetrahedral sites and to the mixed valence $\mathrm{Fe}^{2+} / \mathrm{Fe}^{3+}$ in the octahedral sites of the magnetite. The numerical analysis of these Mössbauer spectra was carried out using the NORMOS ${ }^{\mathrm{TM}}$ software. Similar Debye temperatures were also assumed for both Fe-oxide phases, in order to have their relative areas. Results revealed that the hydrophilic nanoparticles have a composition of $60 \%$ maghemite and $40 \%$ magnetite, whereas the hydrophobic nanoparticles is composed by $53 \%$ maghemite and $47 \%$ magnetite. The lower amount of maghemite in the functionalized nanoparticles with oleic acid is likely due to a protective character of the organic layer, which prevents the magnetite nanoparticle from being oxidized in air.

The adsorption of oleic acid at the external surface of the nanoparticles occurs due to the high affinity for iron minerals. In fact, the negative charge of the carboxyl group is highly attracted to the positive surface of these minerals ${ }^{20,31}$, as schematically shown in Figure 6.

The $\mathrm{pH}$ value at the point of zero charge $\left(\mathrm{pH}_{\mathrm{pzc}}=6.0\right)$ was determined using a Zeta Meter equipment (Figure 7). The 


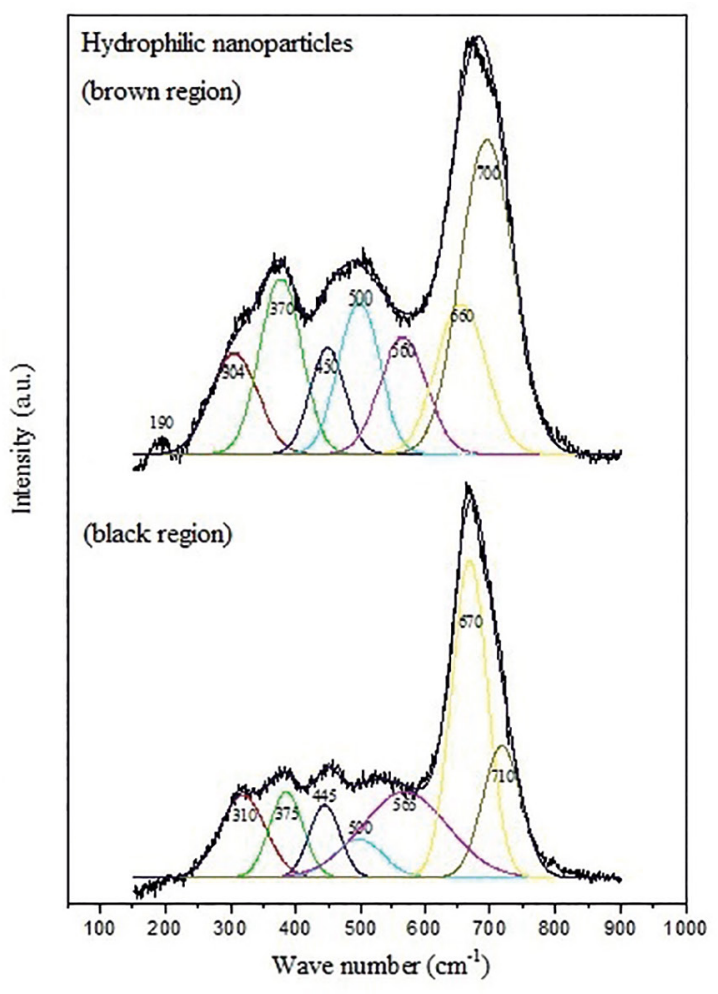

A

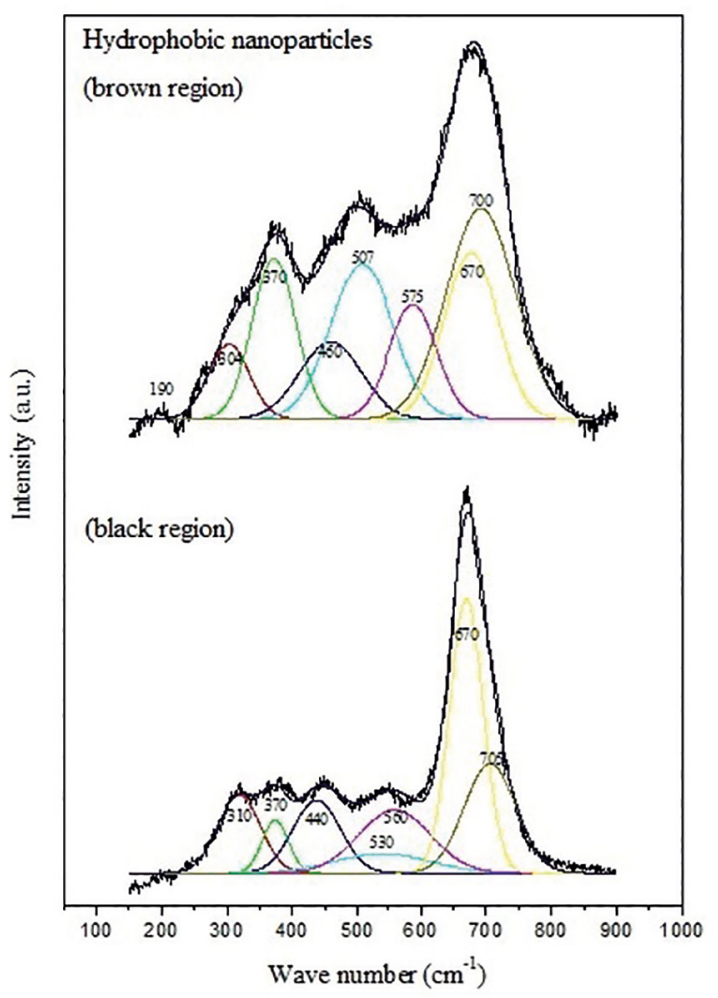

B

Figure 4. Raman spectrum of hydrophilic nanoparticles (A) and the hydrophobic nanoparticles (B), including analysis of the peaks using the Origin software.

Table 1: Vibrational modes of the magnetite and maghemite phases in Raman spectroscopy ${ }^{27-29}$.

\begin{tabular}{lcc}
\hline Phase & Vibrational modes & $\begin{array}{c}\text { Wave number } \\
\left(\mathrm{cm}^{-1}\right)\end{array}$ \\
\hline & $\mathrm{T}_{2 \mathrm{~g}}$ & $190-193$ \\
& $\mathrm{E}_{\mathrm{g}}$ & $306-310$ \\
Magnetite $\left(\mathrm{Fe}_{3} \mathrm{O}_{4}\right)$ & $\mathrm{T}_{2 \mathrm{~g}}$ & $450-490$ \\
& $\mathrm{~T}_{2 \mathrm{~g}}$ & $538-554$ \\
& $\mathrm{~A}_{1 \mathrm{~g}}$ & $668-672$ \\
\hline & $\mathrm{T}_{2 \mathrm{~g}}$ & $350-365$ \\
Maghemite $_{\left(\gamma-\mathrm{Fe}_{2} \mathrm{O}_{3}\right)}$ & $\mathrm{E}_{\mathrm{g}}$ & $500-511$ \\
& $\mathrm{~A}_{1 \mathrm{~g}}$ & 700 \\
\hline
\end{tabular}

isoelectric point of the hydrophilic magnetic nanoparticles found in this work is in fair agreement with the results available in the literature for similar particles, i.e., $5.6^{32}, 6.3^{33}$, and 6.5

22 . Based on this value, the synthesized nanoparticles have a positive charge at $\mathrm{pH}<6$, thus favoring the adsorption of oleic acid molecules. In this study, the adsorption of oleic acid was carried out near $\mathrm{pH} 6$, a value indicated as suitable to obtain the maximum chemisorption of the adsorbent. Moreover, the adsorption process occurred at $60{ }^{\circ} \mathrm{C}$, aimed at favoring the chemical linkage 22,34 .
The Fourier transform infrared spectroscopy analyses confirmed the surface functionalization of magnetite nanoparticles (Figure 8). In relation to the peaks present in both spectra, the $578 \mathrm{~cm}^{-1}$ corresponded to the vibration of the $\mathrm{Fe}-\mathrm{O}$, while the bands 1630 and 3400 $\mathrm{cm}^{-1}$ are characteristic of hydroxyl groups related to the water presence on the surface of the particles ${ }^{19,21}$. In the spectrum of the nanoparticles functionalized with oleic acid (hydrophobic nanoparticles), bands 1425 and 1520 $\mathrm{cm}^{-1}$ are related to the vibration of the carboxyl group (COO-) of oleic acid, while bands 2851 and $2921 \mathrm{~cm}^{-1}$ correspond to the stretching of the $\mathrm{C}-\mathrm{H}$ bonds ${ }^{5,24}$.

The thermograms of the magnetic nanoparticles are shown in Figure 9. The initial weight loss $\left(30^{\circ} \mathrm{C}\right.$ to 120 ${ }^{\circ} \mathrm{C}$ ) is related to the evaporation of water adsorbed on the nanoparticles. The weight loss in this first stage for the hydrophilic nanoparticles was $7.5 \%$, while that for the hydrophobic nanoparticles was much lower, about $0.9 \%$, a value that is coherent with the fact that these particles are hydrophobic. No other relevant modification was attributed to the hydrophilic nanoparticles. On the contrary, the hydrophobic nanoparticles pass through three more weight loss stages. The first two stages, between $120{ }^{\circ} \mathrm{C}$ to $260{ }^{\circ} \mathrm{C}$ and $260{ }^{\circ} \mathrm{C}$ to $450{ }^{\circ} \mathrm{C}$, correspond to the decomposition of the layer of physically adsorbed (3.6\%) 
Table 2: Mössbauer parameters for hydrophilic and hydrophobic magnetic nanoparticles at room temperature and $150 \mathrm{~K}$.

\begin{tabular}{|c|c|c|c|c|c|}
\hline Sample & Phases & $\delta(\mathrm{mm} / \mathrm{s})( \pm 0,05)$ & $\begin{array}{l}\Delta / 2 \xi \mathrm{q}(\mathrm{mm} / \mathrm{s}) \\
( \pm 0,05)\end{array}$ & $\mathrm{B}_{\mathrm{HF}}(\mathrm{T})( \pm 0,2)$ & Area $(\%)( \pm 1)$ \\
\hline \multirow{2}{*}{$\begin{array}{l}\text { Hydrophilic nanoparticles } \\
\text { (RT) }\end{array}$} & $\gamma-\mathrm{Fe}_{2} \mathrm{O}_{3}$ & 0.22 & -0.06 & 47.0 & 38 \\
\hline & $\left(\gamma-\mathrm{Fe}_{2} \mathrm{O}_{3} / \mathrm{Fe}_{3} \mathrm{O}_{4}\right)$ & 0.37 & -0.06 & 41.6 & 62 \\
\hline \multirow{3}{*}{$\begin{array}{l}\text { Hydrophilic nanoparticles } \\
(150 \mathrm{~K})\end{array}$} & $\gamma-\mathrm{Fe}_{2} \mathrm{O}_{3}$ & 0.40 & -0.05 & 50.3 & 60 \\
\hline & $\begin{array}{c}\mathrm{Fe}_{3} \mathrm{O}_{4}\left(\mathrm{Fe}^{3}\right) \\
\text { (tetrahedral site) }\end{array}$ & 0.31 & -0.08 & 48.1 & 14 \\
\hline & $\begin{array}{l}\mathrm{Fe}_{3} \mathrm{O}_{4}\left(\mathrm{Fe}^{3}+\mathrm{Fe}^{2}\right) \\
\text { (octahedral site) }\end{array}$ & 0.60 & -0.02 & 46.4 & 26 \\
\hline \multirow{2}{*}{$\begin{array}{l}\text { Hydrophobic nanoparticles } \\
\text { (RT) }\end{array}$} & $\gamma-\mathrm{Fe}_{2} \mathrm{O}_{3}$ & 0.29 & -0.02 & 46.1 & 31 \\
\hline & $\left(\gamma-\mathrm{Fe}_{2} \mathrm{O}_{3} / \mathrm{Fe}_{3} \mathrm{O}_{4}\right)$ & 0.38 & -0.06 & 41.4 & 61 \\
\hline \multirow{3}{*}{$\begin{array}{l}\text { Hydrophobic nanoparticles } \\
(150 \mathrm{~K})\end{array}$} & $\gamma-\mathrm{Fe}_{2} \mathrm{O}_{3}$ & 0.41 & -0.08 & 50.6 & 53 \\
\hline & $\begin{array}{c}\mathrm{Fe}_{3} \mathrm{O}_{4}\left(\mathrm{Fe}^{3}\right) \\
\text { (tetrahedral site) }\end{array}$ & 0.31 & -0.02 & 48.1 & 16 \\
\hline & $\begin{array}{l}\mathrm{Fe}_{3} \mathrm{O}_{4}\left(\mathrm{Fe}^{3}+\mathrm{Fe}^{2}\right) \\
\text { (octahedral site) }\end{array}$ & 0.64 & -0.02 & 45.4 & 31 \\
\hline
\end{tabular}

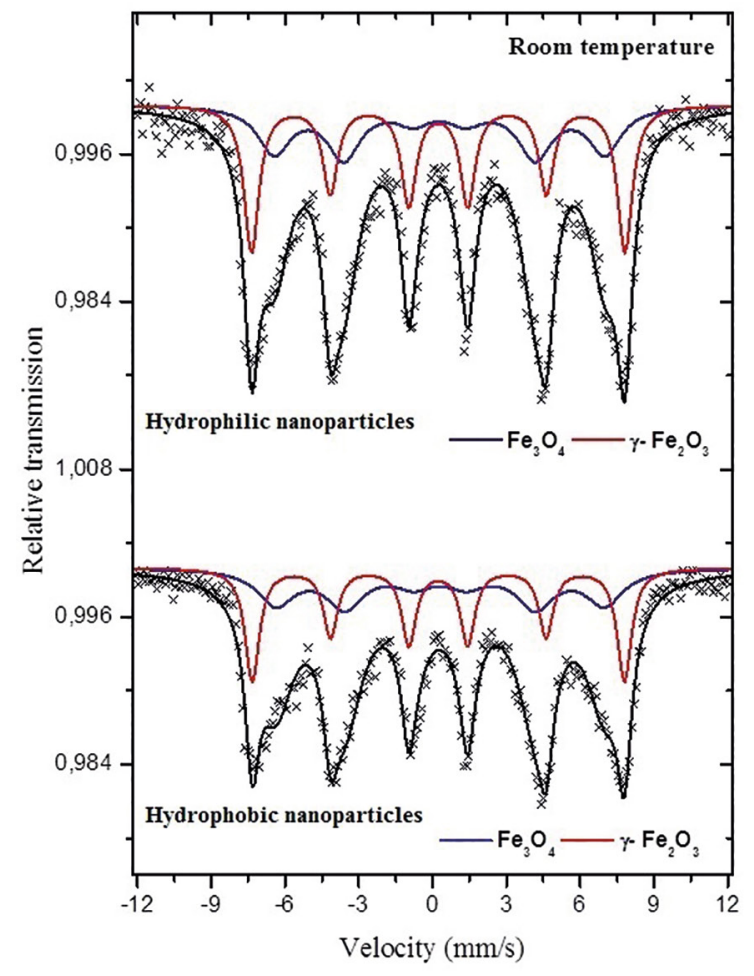

A

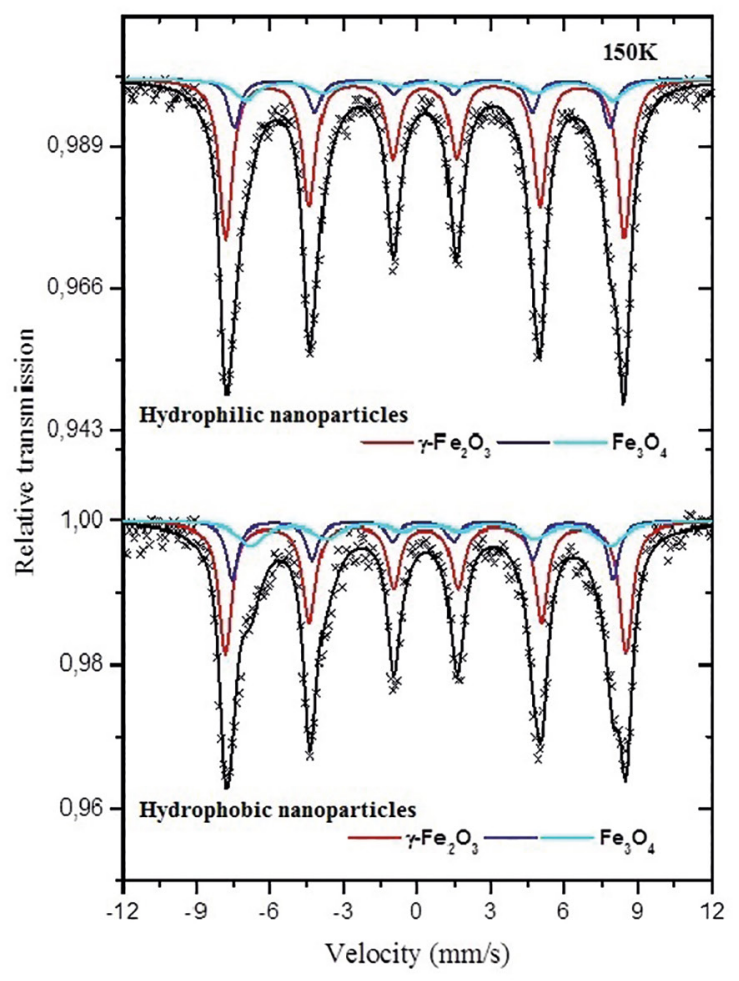

B

Figure 5. Mössbauer spectra of the magnetic nanoparticles at room temperature (A) and $150 \mathrm{~K}$ (B).

and chemically adsorbed (5.6\%) oleic acid, respectively, on the surface of the nanoparticles ${ }^{5,19}$. These layers represent 0.045 and $0.115 \mathrm{~mL}$ of oleic acid adsorbed physically and chemically, respectively, for each gram of magnetic nanoparticles. The third stage of weight loss is above $450{ }^{\circ} \mathrm{C}-500{ }^{\circ} \mathrm{C}$. According to Ayyappan at al. ${ }^{35}$, the degradation of oleic acid produces reduction gases like $\mathrm{CO}$ and $\mathrm{CO}_{2}$, which are responsible for reducing the magnetic nanoparticles. During heating, the possible reduction reactions may occur ${ }^{35}$ :

$$
\begin{gathered}
\mathrm{Fe}_{3} \mathrm{O}_{4}+\mathrm{CO} \longrightarrow 3 \mathrm{FeO}+\mathrm{CO}_{2} \\
3 \mathrm{FeO} \leftrightarrow \mathrm{Fe}_{2} \mathrm{O}_{3}+\alpha \mathrm{Fe} \\
\mathrm{CO}+\frac{1}{2} \mathrm{O}_{2} \leftrightarrow \mathrm{CO}_{2}
\end{gathered}
$$



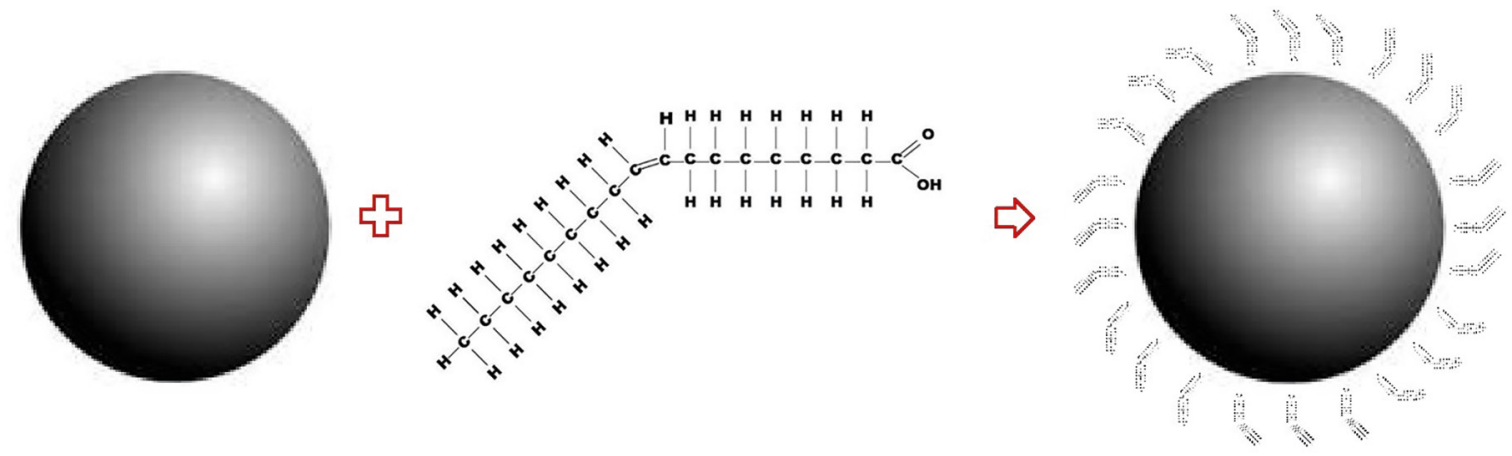

Figure 6. Adsorption of oleic acid on the surface of an iron oxide nanoparticle.

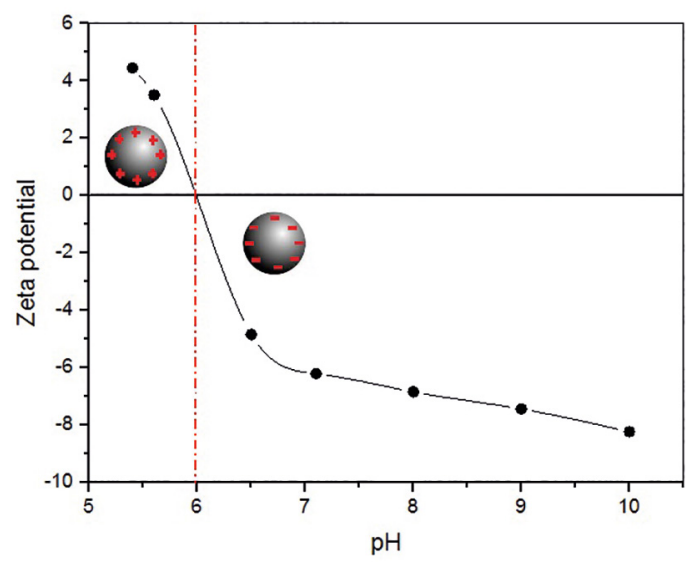

Figure 7. The isoelectric point $\left(\mathrm{pH}_{\mathrm{pzc}}\right)$ of the hydrophilic nanoparticles.

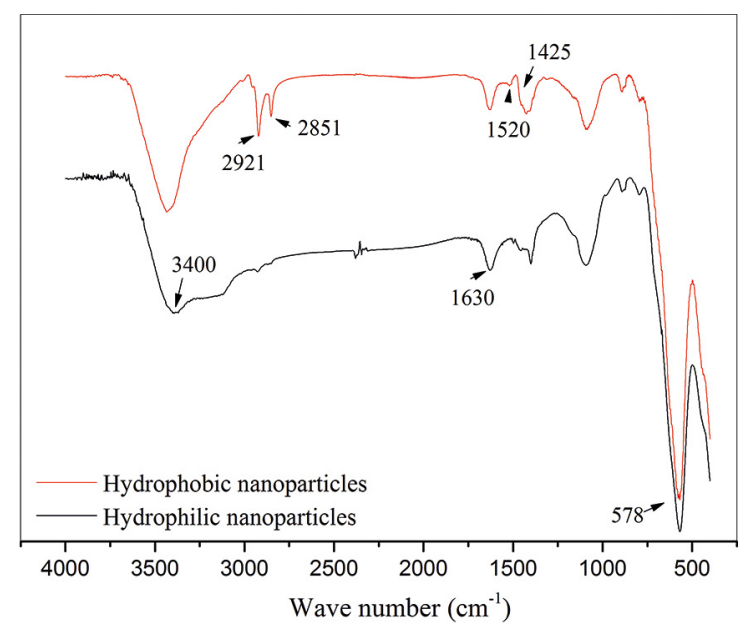

Figure 8. FTIR spectra of the magnetic nanoparticles.

The hysteresis curves of the magnetic particles obtained using a magnetometer are show in Figure 10 - A and the coercivity field values (HC) are show in Figure 10 - B. It can be observed that the samples have a ferro/ferrimagnetic behavior, because the coercive fields at room temperature are non-zero, which is in agreement with the Mössbauer data (six broad lines) above discussed. However, as the coercivity field value is very small, it can be concluded that large part of the

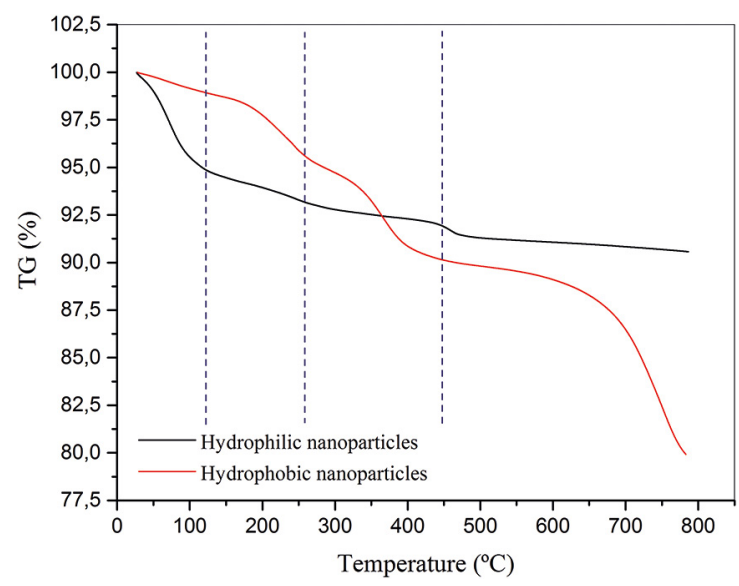

Figure 9. Thermograms of the magnetic nanoparticles.

particles are in the superparamagnetic state. This observation is in agreement with Mössbauer spectroscopy. The values of saturation magnetization and coercivity of the nanoparticles are shown in Table 3. The functionalization with oleic acid did not significantly change the magnetization values. In fact, the small decrease in the magnetization value on the hydrophobic nanoparticle is due to the presence of the organic compound ${ }^{5,23}$ It is noted that the value of the percentage of nonmagnetic material adhered to the magnetic material $(9 \%)$ is almost the same as the magnetization loss between the two samples $(8 \%)$, thus indicating the reason why the nanoparticles functionalized with oleic acid (hydrophobic nanoparticles) lost magnetization.

Considering the fractions of each component (calculated by Mössbauer) and the saturation magnetization values of magnetite bulk phase $\left(92 \mathrm{emu}^{-1}\right)^{-16}$ and maghemite bulk phase $\left(74 \mathrm{emu} \cdot \mathrm{g}^{-1}\right)^{26}$ the nominal magnetization values of the hydrophilic and hydrophobic nanoparticles should be 81.2 and 82.5 emu. $^{-1}$, respectively. These values are higher to those found by magnetic analysis, but in agreement with similar studies reported in the literature ${ }^{23,36}$ This phenomenon can be explained due to the magnetic effects caused by the asymmetric environment of the atoms near to the surface (magnetic deadlayer). As the nanoparticles have a large surface area in relation to their volume, this effect becomes very accentuated ${ }^{37-39}$. 


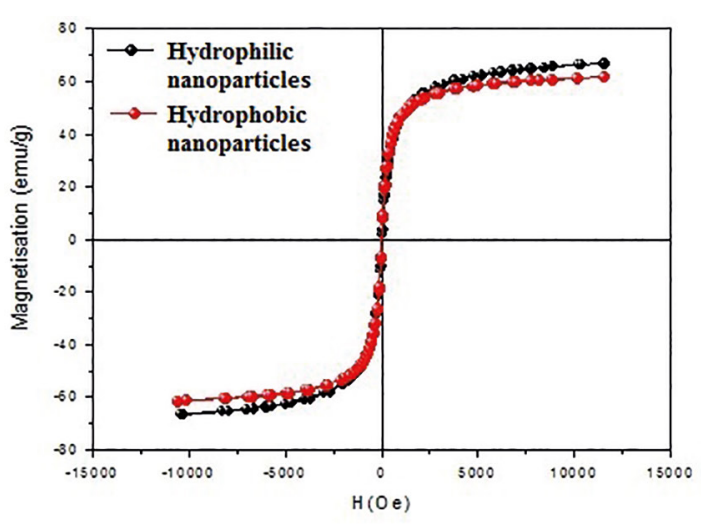

A

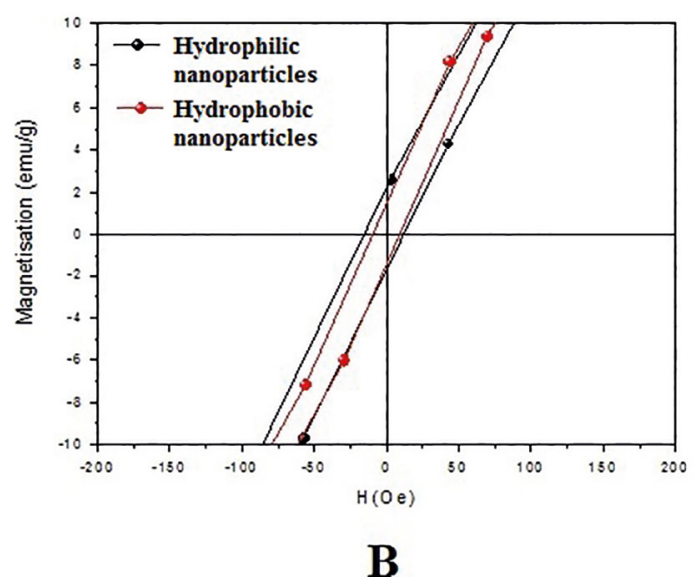

Figure 10. Hysteresis curves (A), and coercivity field values (B) of magnetic nanoparticles.

Table 3: Saturation magnetization and coercivity values of the magnetic nanoparticles.

\begin{tabular}{lcc}
\hline Sample & Coercivity (Gauss) & $\begin{array}{c}\text { Magnetization } \\
(\mathrm{emu} / \mathrm{g})\end{array}$ \\
\hline $\begin{array}{l}\text { Hydrophilic } \\
\text { nanoparticles }\end{array}$ & 13.6 & 66.7 \\
$\begin{array}{l}\text { Hydrophobic } \\
\text { nanoparticles }\end{array}$ & 9.3 & 61.5 \\
\hline
\end{tabular}

The results of the chemical stability of the magnetic nanoparticles are shown in Table 4. Regarding the hydrophilic nanoparticles, no iron was determined in the aqueous solution at $\mathrm{pH} \geq 4$, while the hydrophobic nanoparticles are chemically stable to acid media at $\mathrm{pH} \geq 3$. Furthermore, the loss of iron of the hydrophobic particles is comparatively smaller than that of the hydrophilic nanoparticles when in contact with a medium with the same concentration of $\mathrm{H}^{+}$, which is attributed to the protective layer of oleic acid. The same result of chemical stability is shown in Figure 11 but in relation to the percentage of total iron that contaminated the water. It was observed that while the maximum iron loss of the hydrophobic nanoparticles (functionalized with oleic acid) is $31 \%$, the iron loss in the hydrophilic nanoparticles
Table 4: Iron contamination of the water at changing acidities.

\begin{tabular}{lcc}
\hline Acidity $\left[\mathrm{H}^{+}\right]$ & $\begin{array}{c}\text { Hydrophilic } \\
\text { nanoparticles }\end{array}$ & $\begin{array}{c}\text { Hydrophobic } \\
\text { nanoparticles }\end{array}$ \\
\hline $10^{-7}$ & n.d. & n.d. \\
$10^{-6}$ & n.d. & n.d. \\
$10^{-5}$ & n.d. & n.d. \\
$10^{-4}$ & n.d. & n.d. \\
$10^{-3}$ & $8.2 \pm 0.5$ & n.d. \\
$10^{-2}$ & $83 \pm 1$ & $35.6 \pm 0.4$ \\
$10^{-1}$ & $883 \pm 23$ & $732 \pm 44$ \\
0.5 & $4874 \pm 75$ & $1702 \pm 22$ \\
1.0 & $5578 \pm 2$ & $1807 \pm 36$ \\
2.0 & $5968 \pm 248$ & $2265 \pm 32$ \\
\hline
\end{tabular}

n.d. not detected

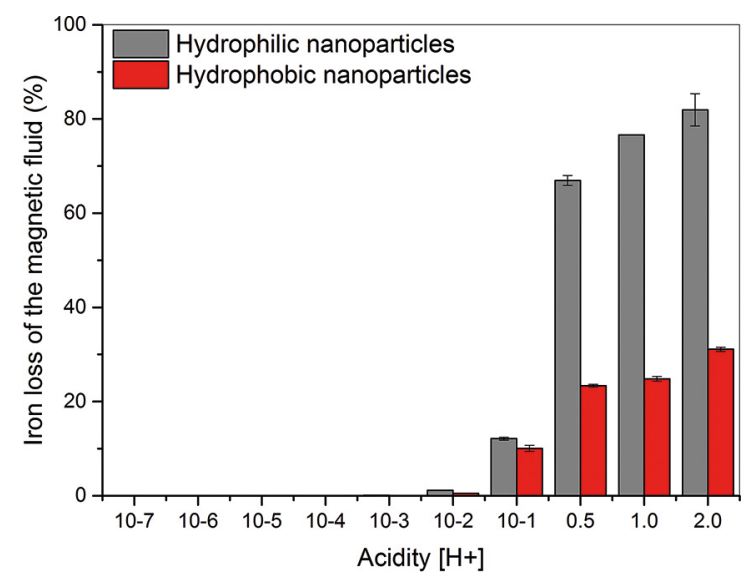

Figure 11. Percentage of iron loss from the magnetic nanoparticles.

reaches $82 \%$. Hence, under appropriate conditions, the magnetic nanoparticles can be employed with no chemical alteration of the environment in which they are applied and with no chemical or morphological alteration of the nanoparticles themselves. Moreover, in suitable conditions, the magnetic nanoparticles have a long service life and can be reused several times.

\section{Conclusions}

The magnetic nanoparticles synthesized through the co-precipitation method (hydrophilic nanoparticles) and subsequently functionalized with oleic acid (hydrophobic nanoparticles) have a spherical shape, with a average nanosize of approximately $10 \mathrm{~nm}$, a large surface area $\left(55-72 \mathrm{~m}^{2} / \mathrm{g}\right)$, a chemical composition of magnetite and maghemite, and a ferro/ferrimagnetic behavior. The hydrophobization by oleic acid, in addition to being an easy, fast, and economical process, ensures a hydrophobic nanoparticle, as well as greater protection against oxidation and acidity of the medium. Moreover, the presence of oleic acid (around 9\% by weight) does not interfere significantly in the saturation 
magnetization value. Regarding chemical stability, the hydrophilic nanoparticles are stable at $\mathrm{pH} \geq 4$, while the hydrophobic nanoparticles are stable at $\mathrm{pH} \geq 3$. It was observed that hydrophobic nanoparticles are relatively more resistant to acid environments than are hydrophilic nanoparticles due to the protection of the organic layer. Thus, under suitable conditions of $\mathrm{pH}$ and in processes involving aqueous and/or organic solutions, the nanoparticles developed in this work can be used for several cycles of the process with no chemical alteration of them or of the environment in which they are.

\section{Acknowledgement}

The authors acknowledge CNPq (n ${ }^{\circ}$ 304050/2016-4), CAPES-PROEX, and FAPEMIG for their financial support, the Center of Microscopy at Universidade Federal de Minas Gerais (UFMG) (http://www.microscopia.ufmg.br) for providing the equipment and technical support for experiments involving electron microscopy, and Centro de Desenvolvimento da Tecnologia Nuclear (CDTN) (http://www.cdtn.br/) for their support with Mössbauer spectroscopy and magnetic analysis.

\section{References}

1. Wang Q, Guan Y, Ren X, Cha G, Yang M. Rapid extraction of low concentration heavy metal ions by magnetic fluids in high gradient magnetic separator. Separation and Purification Technology. 2011;82:185-189.

2. Franzreb M, Siemann-Herzberg M, Hobley TJ, Thomas ORT. Protein purification using magnetic adsorbent particles. Applied Microbiology and Biotechnology. 2006;70(5):505-516.

3. Toma HE. Developing nanotechnological strategies for green industrial processes. Pure and Applied Chemistry. 2013;85(8):1655-1669.

4. Vatta LL, Sanderson RD, Koch KR. Magnetic nanoparticles: Properties and potential applications. Pure and Applied Chemistry. 2006;78(9):1793-1801.

5. Petcharoen K, Sirivat A. Synthesis and characterization of magnetite nanoparticles via the chemical co-precipitation method. Materials Science and Engineering: B. 2012;177(5):421-427.

6. Im SH, Herricks T, Lee YT, Xia Y. Synthesis and characterization of monodisperse silica colloids loaded with superparamagnetic iron oxide nanoparticles. Chemical Physics Letters. 2005;401(13):19-23.

7. Hwang JY, inventor; Board of Control of Michigan Technological University, assignee. Magnetic solvent extraction. United States patent US 5043070 A, 1991 Ago 27.

8. Palyska W, Chmielewski AG. Solvent Extraction and Emulsion Separation in Magnetic Fields. Separation Science and Technology. 1993;28(1-3):127-138.

9. Vatta LL, Koch KR, Sole KC. The potential use of hydrocarbon magnetic liquids in solvent extraction. In: Proceedings of $18^{\text {th }}$ International Solvent Extraction Conference (ISEC 2008); 2008 Sep 15-19; Tucson, AZ, USA. Montreal: Canadian Institute of Mining, Metallurgy and Petroleum; 2008. p. 1513-1518.
10. Lobato NCC, Ferreira AM, Mansur MB. Evaluation of magnetic nanoparticles coated by oleic acid applied to solvent extraction processes. Separation and Purification Technology. 2016;168:93-100.

11. Ge F, Li MM, Ye H, Zhao BX. Effective removal of heavy metal ions $\mathrm{Cd}^{2+}, \mathrm{Zn}^{2+}, \mathrm{Pb}^{2+}, \mathrm{Cu}^{2+}$ from aqueous solution by polymer-modified magnetic nanoparticles. Journal of Hazardous Materials. 2012;211-212:366-372.

12. Silva GC, Almeida FS, Ferreira AM, Ciminelli VST. Preparation and application of a magnetic composite $\left(\mathrm{Mn}_{3} \mathrm{O}_{4} / \mathrm{Fe}_{3} \mathrm{O}_{4}\right)$ for removal of As(III) from aqueous solutions. Materials Research. 2012;15(3):403-408.

13. Yoon SY, Lee CG, Park JA, Kim SB, Kim JH, Lee SH, et al. Kinetic, equilibrium and thermodynamic studies for phosphate adsorption to magnetic iron oxide nanoparticles. Chemical Engineering Journal. 2014;236:341-347.

14. Tang SC, Lo IMC. Magnetic nanoparticles: essential factors for sustainable environmental applications. Water Research. 2013;47(8):2613-2632.

15. Shan C, Ma Z, Tong M. Efficient removal of trace antimony(III) through adsorption by hematite modified magnetic nanoparticles. Journal of Hazardous Materials. 2014;268:229-236.

16. Drenkova-Tuhtan A, Mandel K, Paulus A, Meyer C, Hutter F, Gellermann C, et al. Phosphate recovery from wastewater using engineered superparamagnetic particles modified with layered double hydroxide ion exchangers. Water Research. 2013;47(15):5670-5677.

17. Lee Y, Rho J, Jung B. Preparation of magnetic ion-exchange resins by the suspension polymerization of styrene with magnetite. Journal of Applied Polymer Science. 2003;89(8):2058-2067.

18. Wikström P, Flygare S, Gröndalen A, Larsson PO. Magnetic aqueous two-phase separation: A new technique to increase rate of phase-separation, using dextran-ferrofluid or larger iron oxide particles. Analytical Biochemistry. 1987;167(2):331339.

19. Yang K, Peng H, Wen Y, Li N. Re-examination of characteristic FTIR spectrum of secondary layer in bilayer oleic acidcoated $\mathrm{Fe}_{3} \mathrm{O}_{4}$ nanoparticles. Applied Surface Science. 2010;256(10):3093-3097.

20. Mălăescu I, Gabor L, Claici F, Ştefu N. Study of some magnetic properties of ferrofluids filtered in magnetic field gradient. Journal of Magnetism and Magnetic Materials. 2000;222(1-2):8-12.

21. Cornell RM, Schwertmann U. The Iron Oxides: Structure, Properties, Reactions, Occurrences and Uses. Weinheim: Wiley-VCH Verlag; 2003. 683 p.

22. López-López MT, Durán JDG, Delgado AV, González-Caballero F. Stability and magnetic characterization of oleate-covered magnetite ferrofluids in different nonpolar carriers. Journal of Colloid and Interface Science. 2005;291(1):144-151.

23. Lobaz V, Taylor RNK, Peukert W. Highly magnetizable superparamagnetic colloidal aggregates with narrowed size distribution from ferrofluid emulsion. Journal of Colloid and Interface Science. 2012;374(1):102-110. 
24. Mauricio MR, de Barros HR, Guilherme MR, Radovanovic E, Rubira AF, de Carvalho GM. Synthesis of highly hydrophilic magnetic nanoparticles of $\mathrm{Fe}_{3} \mathrm{O}_{4}$ for potential use in biologic systems. Colloids and Surfaces A: Physicochemical and Engineering Aspects. 2013;417:224-229.

25. Kiss LB, Söderlund J, Niklasson GA, Granqvist CG. New approach to the origin of lognormal size distributions of nanoparticles. Nanotechnology. 1999;10(1):25.

26. Lemine OM, Omri K, Iglesias M, Velasco V, Crespo P, de la Presa $\mathrm{P}$, et al. $\gamma-\mathrm{Fe}_{2} \mathrm{O}_{3}$ by sol-gel with large nanoparticles size for magnetic hyperthermia application. Journal of Alloys and Compounds. 2014;607:125-131.

27. Jubb AM, Allen HC. Vibrational Spectroscopic Characterization of Hematite, Maghemite, and Magnetite Thin Films Produced by Vapor Deposition. ACS Applied Materials \& Interfaces. 2010;2(10):2804-2812.

28. Shebanova ON, Lazor P. Raman spectroscopic study of magnetite $\left(\mathrm{FeFe}_{2} \mathrm{O}_{4}\right)$ : a new assignment for the vibrational spectrum. Journal of Solid State Chemistry. 2003;174(2):424-430.

29. Slavov L, Abrashev MV, Merodiiska T, Gelev C, Vandenberghe RE, Markova-Deneva I, et al. Raman spectroscopy investigation of magnetite nanoparticles in ferrofluids. Journal of Magnetism and Magnetic Materials. 2010;322(14):1904-1911.

30. Gorski CA, Scherer MM. Determination of nanoparticulate magnetite stoichiometry by Mössbauer spectroscopy, acidic dissolution, and powder X-ray diffraction: A critical review. American Mineralogist. 2010;95(7):1017-1026.

31. Soares PIP, Alves AMR, Pereira LCJ, Coutinho JT, Ferreira IMM, Novo CMM, et al. Effects of surfactants on the magnetic properties of iron oxide colloids. Journal of Colloid and Interface Science. 2014;419:46-51.
32. Viota JL, Arroyo FJ, Delgado AV, Horno J. Electrokinetic characterization of magnetite nanoparticles functionalized with amino acids. Journal of Colloid and Interface Science. 2010;344(1):144-149.

33. Vidojkovic S, Rodriguez-Santiago V, Fedkin MV, Wesolowski DJ, Lvov SN. Electrophoretic mobility of magnetite particles in high temperature water. Chemical Engineering Science. 2011;66(18):4029-4035.

34. Peck AS, Raby LH, Wadsworth ME. An Infrared Study of the Flotation of Hematite with Oleic Acid and Sodium Oleate. Transactions of The American Institute of Mining and Metallurgical Engineers. 1967;238:301-307.

35. Ayyappan S, Gnanaprakash G, Panneerselvam G, Antony MP, Philip J. Effect of Surfactant Monolayer on Reduction of $\mathrm{Fe}_{3} \mathrm{O}_{4}$ Nanoparticles under Vacuum. The Journal of Physical Chemistry C. 2008;112(47):18376-18383.

36. Araújo-Neto RP, Silva-Freitas EL, Carvalho JF, Pontes TRF, Silva KL, Damasceno IHM, et al. Monodisperse sodium oleate coated magnetite high susceptibility nanoparticles for hyperthermia applications. Journal of Magnetism and Magnetic Materials. 2014;364:72-79.

37. Iida H, Takayanagi K, Nakanishi T, Osaka T. Synthesis of $\mathrm{Fe}_{3} \mathrm{O}_{4}$ nanoparticles with various sizes and magnetic properties by controlled hydrolysis. Journal of Colloid and Interface Science. 2007;314(1):274-280.

38. Sato T, Iijima T, Seki M, Inagaki N. Magnetic properties of ultrafine ferrite particles. Journal of Magnetism and Magnetic Materials. 1987;65(2-3):252-256.

39. Kim DK, Zhang Y, Voit W, Rao KV, Muhammed M. Synthesis and characterization of surfactant-coated superparamagnetic monodispersed iron oxide nanoparticles. Journal of Magnetism and Magnetic Materials. 2001;225(1-2):30-36. 